\title{
Incidencia de hipotermia perioperatoria inadvertida, en perros sometidos a anestesia general inhalatoria
}

\section{Incidence of inadvertent perioperatory hypothermia, in dogs submitted to inhalation general anesthesia}

\author{
Diego A Ospina-Argüelles'; Edwin Buriticá-Gaviria ${ }^{2}$; Diego Echeverry-Bonilla ${ }^{3}$
}

${ }^{1}$ MVZ, Esp., Pedagogía, M.Sc. Universidad del Tolima, Clínica Médica y Quirúrgica en Pequeños Animales, Grupo de Investigación en Medicina y Cirugía de Pequeños Animales. Ibagué - Tolima, Colombia; e-mail: daospina@ut.edu.co; (1) http://orcid.org/0000-0003-1778-1504

${ }^{2}$ Esp. M.Sc. Universidad del Tolima, Clínica Médica y Quirúrgica en Pequeños Animales, Grupo de Investigación en Medicina y Cirugía de Pequeños Animales. Ibagué - Tolima, Colombia; e-mail: efburiticag@ut.edu.co; (D) http://orcid.org/0000-0002-7979-4135

${ }^{3}$ MVZ, Esp. M.Sc., Ph.D. Universidad del Tolima, Grupo de Investigación en Medicina y Cirugía de Pequeños Animales. Ibagué - Tolima, Colombia; e-mail: decheverry@ut.edu.co; (D) http://orcid.org/0000-0003-2005-5116

Cómo citar: Ospina-Argüelles, D.A.; Buriticá-Gaviria, E.; Echeverry-Bonilla, D. 2021. Incidencia de hipotermia perioperatoria inadvertida, en perros sometidos a anestesia general inhalatoria. Rev. U.D.C.A Act. \& Div. Cient. 24(1):e1830. http://doi.org/10.31910/ rudca.v24.n1.2021.1830

Artículo de acceso abierto publicado por Revista U.D.C.A Actualidad \& Divulgación Científica, bajo una licencia Creative Commons CC BY-NC 4.0

Publicación oficial de la Universidad de Ciencias Aplicadas y Ambientales U.D.C.A, Institución de Educación Superior Acreditada de Alta Calidad por el Ministerio de Educación Nacional.

Recibido: septiembre 13 de 2019 Aceptado: enero 26 de $2021 \quad$ Editado por: Ingeborg Zenner de Polanía

\section{RESUMEN}

La incidencia de hipotermia perioperatoria inadvertida es una de las principales complicaciones perianestésicas en caninos. El objetivo del presente estudio fue evaluar la incidencia de hipotermia perioperatoria inadvertida en 49 perros sometidos a anestesia general inhalatoria. Al finalizar el periodo preanestésico, transanestésico y postanestésico, se registró la temperatura corporal, de acuerdo con los siguientes rangos: hipertermia $\left(>39,5^{\circ} \mathrm{C}\right)$, normotermia $(38,5-$ $\left.39,5^{\circ} \mathrm{C}\right)$, hipotermia leve $\left(38,4-36,5^{\circ} \mathrm{C}\right)$, hipotermia moderada $(36,4-$ $\left.34,0^{\circ} \mathrm{C}\right)$, hipotermia severa $\left(<34^{\circ} \mathrm{C}\right)$. En preanestesia, el $65 \%$ de los animales mostraron hipotermia leve y $2 \%$ hipotermia moderada. En transanestesia, 55\% de los animales tuvieron hipotermia leve, 37\% hipotermia moderada y 6,1\% hipotermia severa. En postanestesia, $59 \%$ de los animales tuvieron hipotermia leve, 29\% hipotermia moderada y $6 \%$ hipotermia severa. Se encontraron diferencias al comparar la temperatura basal, con la final transanestésica y postanestésica, respectivamente $(\mathrm{p}<0,001)$; del mismo modo, se obtuvieron diferencias al comparar la temperatura inicial, con la final del periodo transanestésico $(\mathrm{p}<0,001)$. Por otra parte, no hubo relación estadística entre las diferentes clasificaciones ASA, los procedimientos realizados, el sexo y los rangos etarios evaluados, así como tampoco hubo diferencia en el tiempo de extubación frente al tipo de procedimiento realizado. Los resultados de este estudio sugieren que, bajo las condiciones aquí planteadas, la incidencia de hipotermia preanestésica es mínima, mientras la aparición de hipotermia transanestésica y postanestésica de carácter leve, moderada y severa es mayor.

Palabras clave: Periodo postoperatorio; Periodo preoperatorio; Temperatura; Termorregulación. 


\section{ABSTRACT}

The incidence of inadvertent perioperative hypothermia is one of the main perianesthetic complications in canines. The objective of the present study was to evaluate the incidence of inadvertent perioperative hypothermia in 49 dogs undergoing inhalation general anesthesia. At the end of the pre-anesthetic, trans-anesthetic, and post-anesthetic periods, body temperature was recorded, according to the following ranges: hyperthermia $\left(>39.5^{\circ} \mathrm{C}\right)$, normothermia $\left(38.5-39.5^{\circ} \mathrm{C}\right)$, mild hypothermia $\left(38,4-36.5^{\circ} \mathrm{C}\right)$, moderate hypothermia $\left(36.4-34.0^{\circ} \mathrm{C}\right)$, severe hypothermia $\left(<34^{\circ} \mathrm{C}\right)$. In pre-anesthesia, $65 \%$ of the animals showed mild hypothermia, and $2 \%$ moderate hypothermia. In transanesthesia, $55 \%$ of the animals had mild hypothermia, 37\% moderate hypothermia, and $6 \%$ severe hypothermia. In post-anesthesia $59 \%$ of the animals had mild hypothermia, 29\% moderate hypothermia, and 6\% severe hypothermia. Differences were found when comparing the basal temperature with the final trans-anesthetic and post-anesthetic respectively $(p<0.001)$; Similarly, differences were obtained when comparing the initial temperature with the end of the transanesthetic period $(\mathrm{p}<0.001)$. On the other hand, there was no statistical relationship between the different ASA classifications, the procedures performed, sex, and the evaluated age ranges; as well as there was no difference in the extubation time compared to the type of procedure performed. The results of this study suggest that, under the conditions outlined here, the incidence of pre-anesthetic hypothermia is minimal, while the appearance of mild, moderate, and severe trans-anesthetic and post-anesthetic hypothermia is higher.

Keywords: Post-operative period; Pre-operative period; Temperature; Thermoregulation.

\section{INTRODUCCIÓN}

La temperatura corporal hace parte de los parámetros a monitorizar en el periodo perianestésico en humanos y animales (ÁlvarezReséndiz et al. 2013; Grubb et al. 2020); no obstante, a pesar de estar estipulada en los estándares de registro anestésico, históricamente, ha sido el parámetro menos valorado en el hombre (Castillo et al. 2013; Bindu et al. 2017), por lo que su evaluación en medicina veterinaria podría ser aún menos estricta.

La hipotermia perioperatoria inadvertida (HPI) es definida como la temperatura corporal central $<36^{\circ} \mathrm{C}$ (Riley \& Andrzejowski, 2018). Su incidencia aumenta la morbimortalidad perioperatoria al susceptibilizar los pacientes a la diátesis hemorrágica, incrementar el riesgo de infección, retrasar la cicatrización de heridas, sensibilizar los cardiomiocitos a la aparición de arritmias y alterar la farmacocinética de los medicamentos, entre otros (Hart et al. 2011; Clarke-Price, 2015). Para evitar dichas complicaciones, la HPI puede ser evitada o controlada con estrategias de calentamiento activas o pasivas (ClarkePrice, 2015); sin embargo, es complejo controlar una complicación que no ha sido diagnosticada, previamente, en las condiciones medioambientales de Ibagué.
En medicina humana, la incidencia de HPI es del 25,7-44,3\% (Yang et al. 2015; Yi et al. 2017). En el perro, el panorama no es distinto, encontrándose una incidencia de hipotermia postanestésica leve, moderada y severa, del 51,5, 29,3 y 2,8\%, respectivamente (Redondo et al. 2012). Del conocimiento de los autores de este estudio, Redondo et al. (2012) son los únicos que han descrito, recientemente, la incidencia de esta complicación anestésica en el perro; no obstante, sus hallazgos fueron obtenidos en quirófanos con ambiente controlado, razón por la cual, no son $100 \%$ extrapolables al contexto colombiano, donde no, en todos los quirófanos, se tienen dichas condiciones medioambientales. Por otro lado, los hallazgos anteriormente descritos no esclarecen la incidencia de hipotermia inadvertida preoperatoria y transquirúrgica en el perro, siendo, hasta la fecha, una incógnita del conocimiento. Por tal razón, el objetivo de este estudio fue determinar la incidencia de HPI en perros sometidos a anestesia general inhalatoria.

\section{MATERIALES Y MÉTODOS}

Durante junio de 2017 y junio de 2018, se realizó un estudio clínico prospectivo aleatorizado, avalado por el comité de Bioética de la de la Universidad del Tolima, elaborado bajo los lineamientos de las leyes 84 de 1989 y 1774 de 2016 de protección animal y desarrollado en la Clínica de Pequeños Animales de la Universidad del Tolima, localizada en Ibagué-Tolima, Colombia, a una altura de $1.527 \mathrm{~m}$ s.n.m., presión barométrica de $657 \mathrm{mmHg}$, temperatura ambiente promedio de $23,2^{\circ} \mathrm{C}$ y humedad relativa $65-84 \%$.

Animales. 49 perros de diferentes razas (17 machos y 32 hembras), con un peso de 3,6-48,2kg y una edad de 0,58-17 años fueron incluidos en el estudio, tras diligenciar los dueños el respectivo consentimiento informado. Los pacientes fueron anestesiados por motivos diagnósticos o terapéuticos; solo aquellos con un registro anestésico completo de una duración mayor o igual a 30 minutos, se tuvieron en cuenta. No se emplearon medidas activas de calentamiento transanestésico; únicamente se empleó un aislante térmico para camping (Klimber ${ }^{\circledR}$, Colombia), en la mesa quirúrgica y en el canil de recuperación, al igual que una manta en el postoperatorio inmediato. Durante el estudio, se contó con una temperatura ambiental de $24,94 \pm 2,89^{\circ} \mathrm{C}$ y una humedad relativa de $41,71 \pm 15,61 \%$.

Protocolo anestésico. La fluidoterapia, medicación preanestésica, inducción anestésica y circuito anestésico, fueron seleccionados por un único anestesista, según las consideraciones propias de cada paciente. El mantenimiento anestésico, se realizó con isoflurano, en $\mathrm{O}_{2}$ al 40-100\%.

Variables de estudio. Los siguientes datos fueron considerados:

- Datos de identificación: sexo, edad (años), peso (kg).

- Motivo de la anestesia: procedimientos diagnósticos o quirúrgicos o terapéuticos que requirieran de celiotomía (pe. Laparotomía, ovariohisterectomía, enteroanastomosis, entre otros) y procedimientos diagnósticos o quirúrgicos o terapéuticos, que no requirieran de celiotomía (pe. Detartraje, endoscopia, osteosíntesis, entre otros). 
- Clasificación, de acuerdo con la American Society of Anesthesiologists (ASA): se asignó una clasificación ASA de I-IV, según los hallazgos fisiopatológicos propios de cada paciente. Los pacientes ASA V y ASA E no fueron tenidos en cuenta en la investigación.

- Temperatura preanestésica, transanestésica y postanestésica: la temperatura preanestésica fue medida con un termómetro digital predictivo (Termómetro digital Funny Zoo-Renny ${ }^{\circledR}$, Biomateriales de Venezuela, Estado Miranda, Venezuela), cada 10 minutos hasta el momento de la intubación orotraqueal. La temperatura transanestésica fue medida con un termistor esofágico (IPM 12 VET $^{\circledR}$, Mindray Medical international Limited, Shenzhen, China), cada 10 minutos hasta el momento de la extubación orotraqueal. La temperatura postanestésica fue medida con un termómetro digital predictivo cada 15 minutos hasta completar una hora de registros.

- Temperatura ambiental y humedad relativa: fueron registrados cada 10 minutos, con un termohigrómetro digital (Higrómetro HTC- ${ }^{\circledR}$, Daphne, USA), desde el inicio de los registros termométricos preanestésicos hasta el último registro postoperatorio.

- Temperatura final: Al terminar cada periodo anestésico, la temperatura final de cada paciente, se definió así: hipertermia $\left(>39,5^{\circ} \mathrm{C}\right)$, normotermia $\left(38,5-39,5^{\circ} \mathrm{C}\right)$, hipotermia leve $(38,4-$ $\left.36,5^{\circ} \mathrm{C}\right)$, hipotermia moderada $\left(36,4-34,0^{\circ} \mathrm{C}\right)$, hipotermia severa $\left(<34^{\circ} \mathrm{C}\right)$ (Redondo et al. 2012).

- Duración del procedimiento: se registró en minutos el tiempo preanestésico (desde la aplicación del medicamento tranquilizante/sedante hasta el momento previo a la intubación orotraqueal), tiempo transanestésico (desde el momento de la intubación orotraqueal hasta el momento del cese del agente hipnótico) y tiempo de extubación orotraqueal (desde el momento del cese del agente hipnótico hasta el momento de la extubación orotraqueal).

- Mortalidad anestésica: se llevó registro de los pacientes que murieron en el tiempo comprendido entre la medicación preanestésica y 72 horas posteriores a la extubación.

Análisis estadístico. Los datos fueron tabulados y analizados mediante estadística descriptiva, expresada como la media \pm desviación estándar (DE). Para determinar la normalidad de los datos, se empleó el test de Kolmogorov-Smirnov. Los datos con distribución normal fueron analizados por medio de análisis de varianza de una vía (ANOVA) y un posterior análisis de Tukey, mientras los no paramétricos fueron analizados, mediante prueba de Kruskal-Wallis, seguida del test exacto de Fisher. Los valores a lo largo del tiempo fueron evaluados, mediante prueba de comparaciones múltiples de Dunn's. Se realizaron correlaciones estadísticas, cumpliendo los supuestos de normalidad, para coeficiente $\mathrm{p}$ de Pearson y de no normalidad para Rho de Spearman $(\mathrm{p}<0,05)$. Los datos, se analizaron con un nivel de confianza del $95 \%$, en el paquete estadístico GraphPad Prism 6.0, California, USA.

\section{RESULTADOS Y DISCUSIÓN}

Del conocimiento de los autores, este es el primer estudio que describe la incidencia de HPI en perros anestesiados. Los resultados de esta investigación evidencian una disminución significativa de la temperatura en el periodo transanestésico y postanestésico frente a sus valores basales y muestra una tendencia clínica hacia la hipotermia, en el periodo preanestésico (Tabla 1).

El estudio fue desarrollado sin complicaciones, a excepción de ocho perros, que fueron retirados de la investigación, por ser sometidos a procedimientos inferiores a treinta minutos, al igual que 5 animales anestesiados fuera del quirófano, que no tuvieron un registro anestésico completo. Ningún animal murió en el periodo perianestésico, ni en un lapso de hasta 72 horas, posteriores al procedimiento.

De los animales evaluados, 11 fueron ASA I, 23 ASA II, 13 ASA III, y 2 ASA IV. 22 animales (44,9\%) fueron sometidos a procedimientos que requirieron celiotomía y $27(55,1 \%)$, a procedimientos que no la requirieron (Tabla 2). La duración promedio del tiempo preanestésico, transanestésico y postanestésico fue $31,34 \pm 11,91 \mathrm{~min}$

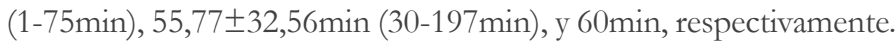
Los valores de humedad relativa y temperatura ambiental fueron $55,19 \pm 17,69 \%$ y $24,29 \pm 1,13^{\circ} \mathrm{C}$ (preanestesia), $37,43 \pm 10,49 \%$ y $24,01 \pm 2,67^{\circ} \mathrm{C}$ (transanestesia), y $36,24 \pm 13,06 \%$ y $26,65 \pm 3,44^{\circ} \mathrm{C}$ (postanestesia).

La temperatura corporal basal promedio fue $38,36 \pm 0,6^{\circ} \mathrm{C}$, similar a lo reportado para la especie (Clarke et al. 2014; Thomas \& Lerche, 2017). Los valores finales de temperatura corporal en los distintos periodos fueron $38,08 \pm 0,8^{\circ} \mathrm{C}$ (preanestesia), $36,19 \pm 1,1^{\circ} \mathrm{C}$ (transanestesia), y $36,66 \pm 1,2^{\circ} \mathrm{C}$ (postanestesia). El valor final de temperatura

Tabla 1. Contrastes entre los valores de temperatura corporal basal, temperatura corporal preanestésica final, temperatura corporal transanestésica final y temperatura corporal postanestésica final, de 49 perros sometidos a anestesia general inhalatoria.

\begin{tabular}{|c|c|}
\hline Contrastes & Significancia \\
\hline $\mathrm{T}^{\circ} \mathrm{C}$ basal $v$ s $\mathrm{T}^{\mathrm{o}}$ preanestésica final & 0,09 \\
\hline $\mathrm{T}^{\circ} \mathrm{C}$ basal $v s \mathrm{~T}^{\mathrm{o}}$ transanestésica final & $<0,001$ \\
\hline $\mathrm{T}^{\circ} \mathrm{C}$ basal $v$ s $\mathrm{T}^{\mathrm{o}}$ postanestésica final & $<0,001$ \\
\hline
\end{tabular}

$\mathrm{T}^{\circ} \mathrm{C}$ : Temperatura corporal 
Tabla 2. Distribución de los animales sometidos a procedimientos que requirieron celiotomía y procedimientos que no requirieron celiotomía, en 49 perros sometidos a anestesia general inhalatoria.

\begin{tabular}{|c|c|c|c|}
\hline \multicolumn{2}{|c|}{ Procedimientos que requirieron celiotomía } & \multicolumn{2}{c|}{ Procedimientos que no requirieron celiotomía } \\
\hline Procedimiento & $\mathrm{N}^{\text {o de animales }}$ & Procedimiento & $\mathrm{N}^{\text {o de animales }}$ \\
\hline Ovariohisterectomía electiva & 13 & Mastectomía & 4 \\
\hline Ovariohisterectomía facultativa & 6 & Detartraje & 5 \\
\hline Enteroanastomosis & 2 & Lumpectomía & 1 \\
\hline Cistotomía & 1 & Biopsia excisional & 1 \\
\hline & & Orquiectomía & 2 \\
\hline & & $\begin{array}{c}\text { Corrección de prolapso } \\
\text { de tercer parpado }\end{array}$ & 1 \\
\hline & & Endoscopia & 4 \\
\hline & & $\begin{array}{c}\text { Osteosíntesis con placa } \\
\text { Ablación de cabeza y } \\
\text { cuello femoral }\end{array}$ & 5 \\
\hline & & $\begin{array}{c}\text { Amputación mediofemo- } \\
\text { ral }\end{array}$ & 3 \\
\hline
\end{tabular}

corporal preanestésica, se encontró en el rango fisiológico (Clarke et al. 2014; Thomas \& Lerche, 2017). Diferentemente, los valores finales de temperatura transanestésica y postanestésica fueron relacionados con cuadros de hipotermia inadvertida (Tabla 3), la cual, tradicionalmente, ha sido clasificada como hipotermia leve $\left(37-32^{\circ} \mathrm{C}\right)$, hipotermia moderada $\left(32-28^{\circ} \mathrm{C}\right)$, e hipotermia severa $\left(<28^{\circ} \mathrm{C}\right)$ (Ortega, 2016). Dichos rangos no fueron contemplados fielmente en el presente estudio, ya que, de haberlo hecho los valores finales de temperatura transanestésica y postanestésica, habrían sido catalogados solo como eventos de hipotermia leve; por tal razón, para realizar el análisis de datos con un modelo más bondadoso, se implementaron los rangos de hipotermia, planteados por Redondo et al. (2012), permitiendo clasificar los valores finales de temperatura transanestésica en hipotermia moderada y los valores finales de temperatura postanestésica, en hipotermia leve (Tabla 3).

En la tabla 4 se observa el comportamiento preanestésico de la temperatura central en los animales evaluados. No se encontraron diferencias significativas entre la temperatura rectal basal frente a la temperatura final preanestésica ( $\mathrm{p}>0,05)$ (Tabla 1); sin embargo, se observó una tendencia clínica hacia la hipotermia en el periodo preanestésico evaluado (Tabla 3). Para simplificar el modelo, en el presente estudio, no se contempló la relación de los fármacos preanestésicos empleados sobre la temperatura corporal; sin embargo, se debe mencionar que un fármaco tranquilizante/ sedante al disminuir los niveles de ansiedad, el estrés, el gasto energético, el gasto cardiaco, entre otros, puede afectar también el comportamiento de la temperatura corporal, haciéndola disminuir (Pottie et al. 2007; Pawson, 2008; Clarke et al. 2014), tal como lo describen los resultados obtenidos. Por otro lado, se debe resaltar que dichos cambios son dosis dependientes y que no siempre se suelen presentar de igual manera en todos los animales, ya que influyen factores, como la idiosincrasia, peso corporal, superficie de área corporal, grasa corporal, condición corporal, edad, entre otros (Brodeur et al. 2017). Por otro lado, se ha descrito que factores, como la obesidad, disminuyen la incidencia de hipotermia, al minimizar la conductividad de calor del individuo al medio externo (Fernandes et al. 2012). Lo mencionado explica por qué un individuo presentó un cuadro de hipertermia en el periodo preanestésico evaluado, ya que tenía una condición corporal 5/5.

Se han descrito 3 fases en la aparición de HPI: la primera es la fase exponencial, donde se produce una rápida disminución inicial de la temperatura, producto de la redistribución calórica, con pérdida entre $1,0-1,5^{\circ} \mathrm{C}$, durante la primera hora de anestesia. La segunda es la fase lineal, donde hay una disminución lenta de la temperatura central, producida entre 2-3 horas, lo cual, se debe a que la pérdida de calor excede la tasa de producción calórica metabólica. Por último, en la fase de meseta, se detiene la pérdida de calor después 3-5 horas de la aparición de la HPI, ya que la pérdida de calor es igual a la producción del mismo (Hart et al. 2011; Muir et al. 2013). Esto concuerda, con lo descrito en la literatura (Redondo et al. 2012; Grimm, 2015) y con lo observado en la figura 1, donde se evaluó el comportamiento de la temperatura corporal a lo largo de 200 minutos de monitorización anestésica.

$\mathrm{Al}$ correlacionar la temperatura transanestésica final con diferentes rangos de tiempo quirúrgico ( $<1$ hora, 2-3 horas, $>3$ horas), no se encontraron diferencias significativas, contrario a lo que habría de esperarse ( $\mathrm{p}>0,05)$, ya que, como se observó en la figura 1 , hay una relación inversa entre el tiempo anestésico y la temperatura corporal, es decir, que a mayor tiempo quirúrgico la temperatura corporal va a ser menor. Esto, se pudo deber al bajo número de animales, cuya anestesia superó las 2 horas de monitorización, ya 
Tabla 3. Valores iniciales y finales de la temperatura en el periodo perianestésico, de perros anestesiados bajo anestesia general inhalatoria.

\begin{tabular}{|c|c|c|c|}
\hline Grupos & Inicial & Final & Significancia \\
\hline Basal & $38,36 \pm 0,6{ }^{\circ} \mathrm{C}$ & $(--)$ & $(---)$ \\
\hline Preanestésia & $38,33 \pm 0,6{ }^{\circ} \mathrm{C}$ & $38,08 \pm 0,8^{\circ} \mathrm{C}$ & 0,09 \\
\hline Transanestésia & $37,54 \pm 0,7{ }^{\circ} \mathrm{C}$ & $36,19 \pm 1,1^{\circ} \mathrm{C}$ & $<0,0001$ \\
\hline Postanestésia & $36,32 \pm 1,4^{\circ} \mathrm{C}$ & $36,66 \pm 1,2^{\circ} \mathrm{C}$ & 0,22 \\
\hline
\end{tabular}

${ }^{\circ} \mathrm{C}:$ Grados centígrados

Tabla 4. Incidencia de hipertermia, normotermia e hipotermia, en 49 perros sometidos a anestesia general inhalatoria.

\begin{tabular}{|l|l|l|l|}
\hline & Hipertermia & Normotermia & Hipotermia \\
\hline Preanestesia & $1 / 49(2 \%)$ & $15 / 49(31 \%)$ & $\begin{array}{l}33 / 49(67 \%) \\
\text { Lev 32/49(65\%) } \\
\text { Mod 1/49(2\%) } \\
\text { Sev }(---)\end{array}$ \\
\hline Transanestesia & & 48/49(98\%) & $\begin{array}{l}\text { Lev 27/49(55\%) } \\
\text { Mod 18/49(37\%) } \\
\text { Sev 3/49(6\%) }\end{array}$ \\
\hline Postanestesia & $(---)$ & $1 / 49(2 \%)$ & $\begin{array}{l}46 / 49(94 \%) \\
\operatorname{Lev} 29 / 49(59 \%) \\
\operatorname{Mod} 14 / 49(29 \%) \\
\text { Sev 3/49(6\%) }\end{array}$ \\
\hline
\end{tabular}

Lev: Leve, Mod: Moderada, Sev: Severa

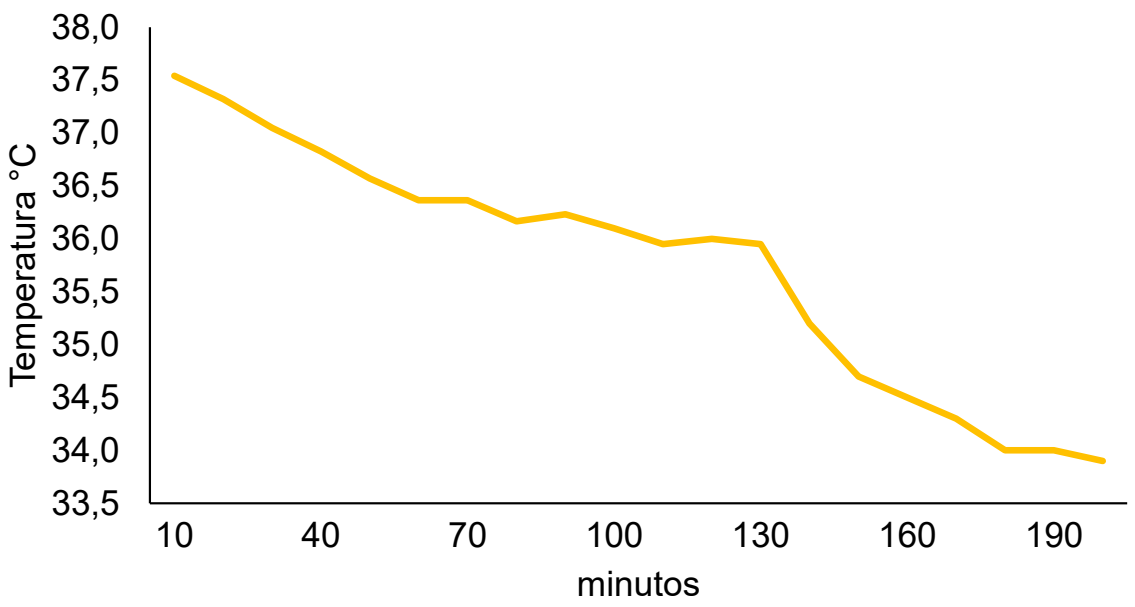

Figura 1. Disminución paulatina de la temperatura esofágica transanestésica a lo largo de 200 minutos de monitorización continua. 
que solo un individuo superó los 180 minutos de anestesia, en el presente estudio.

En la tabla 4, se observa el comportamiento transanestésico de la temperatura en los animales evaluados. Asimismo, la tabla 3, evidencia las diferencias entre la temperatura basal y la de fin de anestesia $(\mathrm{p}<0,05)$. En la tabla 1 , se comparan los valores iniciales y finales de la temperatura perianestésica, encontrando una disminución significativa en el valor final de la temperatura transquirúrgica $(\mathrm{p}<0,001)$.

La disminución de la temperatura perioperatoria observada, se conoce como hipotermia secundaria y es consecuencia directa del acto anestésico o quirúrgico per se (Ortega, 2016; Brodeur et al. 2017). Diversos trabajos han descrito esta misma complicación en el perro (Pottie et al. 2007; Stepaniuk \& Brock, 2008; Redondo et al. 2012). La pérdida de calor perioperatoria, se produce por 4 métodos: radiación, convección, evaporación y conducción (Bindu et al. 2017), siendo la radiación el más relevante, ya que genera hasta el $60 \%$ de la pérdida de calor transanestésico (Duke, 2016); no obstante, anestésicos, como el isoflurano empleado en este estudio, también pueden jugar un rol importante en la pérdida de calor, debido a la vasodilatación periférica que ocasionan, alterando el gradiente de temperatura centro-periférico (Grimm, 2015; Buriticá et al. 2019), lo cual, facilita la pérdida final de calor por convección y conducción (Clark-Price, 2015; Grimm, 2015; Duke, 2016). Por otro lado, hipnóticos, como el Propofol, al inhibir la termogénesis no tiritante, también pueden facilitar las pérdidas de calor, por los métodos anteriormente descritos, empeorando el cuadro clínico del paciente (Bindu et al. 2017).

No se encontraron diferencias significativas al comparar la temperatura final de los pacientes sometidos a procedimientos que requirieron celiotomía frente a la temperatura final, de los que no la requirieron $(35,95 \pm 1,0$ frente a $36,32 \pm 1,1, p=0,238)$; no obstante, se observó una tendencia clínica de mayor pérdida de calor en la realización de procedimientos quirúrgicos que impliquen una laparotomía, lo cual, concuerda con lo reportado en otras investigaciones (Stepaniuk \& Brook, 2008; Redondo et al. 2012; Aarnes et al. 2017), lo cual, se debe a que las pérdidas de calor por evaporación y por radiación son mayores al exponer la cavidad abdominal (Duke, 2016; Thomas \& Lerche, 2017).

En la tabla 4, se observa el comportamiento postanestésico de la temperatura en los animales evaluados. La tabla 3 muestra diferencias significativas entre la temperatura basal y final del periodo postanestésico $(\mathrm{p}<0,05)$. En humanos, se demostró que una temperatura ambiente superior a $23^{\circ} \mathrm{C}$, se puede considerar como un factor protector, al favorecer la ganancia de calor postanestésica (Biazzotto et al. 2006). Contrariamente, en el perro, se describió que temperaturas del área de recuperación inferiores a $27^{\circ} \mathrm{C}$, similar a la registrada en este estudio, favorecen la presencia de hipotermia postoperatoria (Dale et al. 1968); no obstante, se debe resaltar que incrementos en la temperatura perianestésicas aumentan el riesgo de infección perioperatoria, según la experiencia de los autores. Investigaciones recientes abren una nueva brecha de discusión, al defender el uso de temperaturas ambientales transquirúrgicas de $24^{\circ} \mathrm{C}$ (como las obtenidas en el presente estudio), junto a estrategias de calentamiento activas, buscando minimizar la incidencia de hipotermia perioperatoria en perros y en gatos, sometidos a procedimientos quirúrgicos de cavidad abdominal abierta (Rodriguez-Diaz et al. 2019). Otro aspecto importante del área quirúrgica a considerar es la humedad relativa, ya que su disminución favorece la pérdida de calor por evaporación, razón por la cual, se recomiendan niveles de humedad relativa superiores a 45\% (Castillo et al. 2013), similar a los valores registrados en este estudio.

Los contrastes realizados entre las distintas clasificaciones ASA y las temperaturas perianestésicas finales, no arrojaron diferencias significativas (clasificación ASA frente al valor final de temperatura preanestésica final $\mathrm{p}=-0,380$; clasificación ASA frente al valor final de temperatura transanestésica final $\mathrm{p}=0,238$; clasificación ASA frente al valor final de temperatura postanestésica final $\mathrm{p}=$ 0,132); dichos hallazgos contrarían lo reportado en la literatura, ya que en perros anestesiados, se ha establecido una relación directa entre la clasificación ASA y el grado de hipotermia observada (Redondo et al. 2012; Portier \& Ida, 2018). Si bien es cierto, la incidencia de hipotermia en perros aumenta 2,34 veces en pacientes con clasificación ASA > III (Portier \& Ida, 2018), la ausencia de variaciones significativas de temperatura en el presente estudio, se pudo deber al número reducido de individuos con clasificación ASA III y ASA IV analizados, ya que tan solo fueron estudiados 12 y 2 animales de cada grupo, respectivamente, representando tan solo $28,5 \%$ de la población evaluada. Por otro lado, los resultados obtenidos no mostraron cambios relevantes con respecto al género como predictor de hipotermia perioperatoria $(\mathrm{p}>0,05)$, similar a lo reportado por Redondo et al. (2012).

Otro aspecto a tener en cuenta es la edad del paciente, ya que es considerada como un factor de riesgo para la aparición de HPI, debido a que los pacientes muy jóvenes o geriátricos tienen alterada su función termorreguladora (Thomas \& Lerche, 2017); no obstante, a pesar de realizar comparaciones entre diferentes grupos etarios (<1 año, 1-8 años, $>8$ años), con las distintas temperaturas perianestésicas finales, no se encontraron diferencias importantes ( $>>0,05)$, similar a lo reportado en otra investigación (Redondo et al. 2012), lo cual, descartó la influencia de la edad como posible factor predisponente de HPI; sin embargo, al correlacionar las distintas edades con los valores finales de temperatura transanestésica, se encontró una correlación positiva en los animales menores a 1 año $(16,65 \%)$, al igual que para los animales entre 2 y 8 años $(11,53 \%)$, lo que sugiere una correlación positiva con tendencia a disminuir conforme son mayores los grupos etarios, es decir, entre más joven es el animal mayor será la correlación con la temperatura; contrariamente, entre más viejo sea la correlación será menor (Hart et al. 2011; Sanjuán et al. 2011).

Para los individuos mayores a 8 años, la correlación también fue positiva $(0,56 \%)$, aunque se redujo considerablemente, indicando que se debe tener especial precaución al anestesiar este tipo de pacientes, ya que son más propensos a la HPI, por tener un menor metabolismo basal y una respuesta vasoconstrictora periférica menos 
eficaz, comparada con la que tienen los individuos más jóvenes (Sanjuán et al. 2011).

Finalmente, el tiempo promedio de extubación fue 10,88 $\pm 3,55 \mathrm{~min}$, similar a lo reportado por otros autores (Redondo et al. 2012; Aarnes et al. 2017). A pesar de no encontrar diferencias significativas entre los grupos $(p=0,14)$, se observó una tendencia clínica de mayor tiempo de extubación en pacientes sometidos a procedimientos que requirieron laparotomía frente a los animales que no la requirieron $(11,77 \pm 3,02$ frente a $10,15 \pm 3,82 \mathrm{~min})$, lo cual, se debe a que, al tener una mayor pérdida de calor, los animales sometidos a celiotomía pudieron ser más susceptibles al efecto depresor del sistema nervioso central de los fármacos anestésicos (Hart et al. 2011; Clarke-Price, 2015), predisponiéndolos a tiempos de recuperación anestésica más prolongados (Pottie et al. 2007).

Se sugiere realizar estudios con un mayor número de animales en diferentes procedimientos, tanto anestésicos como quirúrgicos; asimismo, contemplar factores, como la superficie de área corporal, índice de masa corporal, índice de grasa corporal, junto a distintos rangos de temperatura ambiental. Por otra parte, se recomienda considerar el efecto de la medicación preanestésica, la fluidoterapia y los diferentes circuitos anestésicos empleados, sobre el comportamiento de la temperatura corporal perioperatoria. Por último, se recomienda realizar evaluaciones termográficas perianestésicas, que permitan esclarecer la dinámica de la HPI en el perro.

Los resultados del presente estudio sugieren que los perros anestesiados con protocolos de anestesia inhalada, bajo las condiciones aquí planteadas, tienen una tendencia clínica a la hipotermia leve en el periodo preanestésico, mientras que la incidencia de hipotermia leve, moderada y severa transanestésica y postanestésica, es alta. La importancia clínica del presente trabajo radica en establecer un punto de partida para afrontar una complicación real, sin previo diagnóstico en Ibagué, permitiendo una mejor toma de decisiones preventivas y correctivas, según fuera el caso.

Conflicto de intereses: El manuscrito fue preparado y revisado con la participación de todos los autores, quienes declaramos que no existe ningún conflicto de intereses que ponga en riesgo la validez de los resultados presentados. Financiación: Este estudio fue financiado por la Universidad del Tolima.

\section{REFERENCIAS}

1. AARNES, T.K.; BEDNARSKI, R.M.; LERCHE, P.; HUBBELL, J.A.E. 2017. Effect of pre-warming on perioperative hypothermia and anesthetic recovery in small breed dogs undergoing ovariohysterectomy. Can. Vet. J. 58(2):175-179.

2. ÁLVAREZ-RESÉNDIZ, G.E.; OCHOA-GAITÁN, G.; VELAZCO-GONZÁLEZ, J.G.; GUTIÉRREZ-PORRAS, C.L.; MONARES-ZEPEDA, E. 2013. Monitoreo anestésico básico. Rev. Mex. Anest. 36(Supl 1):s95-s100.
3. BIAZZOTTO, C.B.; BRUDNIEWSKI, M.; SCHMIDT, A.P.; JUNIOR, J.O.C.A. 2006. Hipotermia no periodo perioperatório. Rev. Bras. Anestesiol. 56(1):89-106. http://doi.org/10.1590/S0034-70942006000100012

4. BINDU, B.; BINDRA, A.; RATH, G. 2017. Temperature management under general anesthesia: Compulsion or option. J. Anaesthesiol. Clin. Pharmacol. (India). 33(3):306316.

https://doi.org/10.4103/joacp.JOACP_334_16

5. BRODEUR, A.; WRIGHT, A.; CORTES, Y. 2017. Hypothermia and targeted temperature management in cats and dogs. J. Vet. Emerg. Crit. Care. (Reino Unido). 27(2):151-163. https://doi.org/10.1111/vec.12572

6. BURITICÁ, E.; ECHEVERRY, D.; OSPINA-ARGÜELLES, D.A. 2019. Efecto de dos maniobras cíclicas de reclutamiento alveolar sobre diversos parámetros de monitoreo microcirculatorio no invasivo en perros. Rev. U.D.C.A Act. \& Div. Cient. (Colombia). 22(1):e1150. https://doi.org/10.31910/rudca.v22.n1.2019.1150

7. CASTILLO, C.G.; CANDIA, C.A.; MARROQUÍN, H.A.; AGUILAR, F.; BENAVIDES, J.J.; ÁLVAREZ, J.A. 2013. Manejo de la temperatura en el perioperatorio y frecuencia de hipotermia inadvertida en un hospital general. Rev. Colomb. Anestesiol. 41(2):97-103. https://doi.org/10.1016/j.rca.2013.03.002

8. CLARK-PRICE，S. 2015. Inadvertent perianesthetic hypothermia in small animal patients. Vet. Clin. North. Am. Small Anim. Pract. (Estados Unidos). 45(5):983-994. https://doi.org/10.1016/j.cvsm.2015.04.005

9. CLARKE, K.W.; TRIM, C.M.; HALL, L.W. 2014. Veterinary anesthesia. Ed. Saunders Elsevier (China). 694p.

10. DALE, H.E.; ELEFSON, E.E.; NIEMEYER, K.H. 1968. Influence of environmental temperature on recovery of dogs from pentobarbital anaesthesia. Am. J. Vet. Res. (Estados Unidos). 29(7):1339-1347.

11. DUKE, J.C. 2016. Temperature disturbances. En: Duke, J.C.; Keech, B.M. (Eds). Duke's anesthesia secrets. Ed. Saunders Elsevier (Estados Unidos). p.177-180.

12. FERNANDES, L.A.; BRAZ, L.G.; KOGA, F.A.; KAKUDA, C.M.; MÓDOLO, N.S.; DE CARVALHO, L.R.; VIANNA, P.T.; BRAZ, J.R. 2012. Comparison of peri-operative core temperature in obese and non-obese patients. Anesthesia (Estados Unidos). 67(12):1364-1369. https://doi.org/10.1111/anae.12002.x 
13. GRIMM, K.A. 2015. Perioperative thermoregulation and heat balance. En: GRIMM, K.A.; LAMONT, L.A.; TRANQUILLI, W.J.; GREENE, S.A.; ROBERTSON, S.A. (Eds). Veterinary anesthesia and analgesia -the fifth edition of Lumb and Jones. Ed. Wiley BlackWell (Estados Unidos). p.372-379.

14. GRUBB, T.; SAGER, J.; GAYNOR, J.S.; MONTGOMERY, E.; PARKER, J.A.; SHAFFORD, H.; TEARNEY, C. 2020. 2020 AAHA Anesthesia and Monitoring Guidelines for Dogs and Cats. J. Am. Anim. Hosp. Assoc. 56(2):59-82. https://doi.org/10.5326/JAAHA-MS-7055

15. HART, S.R.; BORDES, B.; HART, J.; CORSINO, D.; HARMON, D. 2011. Unintended perioperative hypothermia. Ochsner J. (Estados Unidos). 11(3):259-270.

16. MUIR, W.W.; HUBBELL, J.A.E.; BEDNARSKI, R.M.; LERCHE, P. 2013. Handbook of veterinary anesthesia. Ed. Elsevier Mosby (Estados Unidos). 600p.

17. ORTEGA, R.M. 2016. Hipotermia perioperatoria y su riesgo en el paciente quirúrgico. Vanguardia Veterinaria (México). 76:22-26.

18. PAWSON, P. 2008. Sedatives. En: Maddison, J.E.; Page, S.W.; Church, D.B. (eds). Small animal clinical pharmacology. Ed. Saunders Elsevier (China). p.113-125.

19. PORTIER, K.; IDA, K.K. 2018. The ASA physical status classification: what is the evidence for recommending its use in veterinary anesthesia?-A systematic review. Front. Vet. Sci. (Suiza). 5:204.

https://doi.org/10.3389/fvets.2018.00204

20. POTTIE, R.G.; DART, C.M.; PERKINS, N.R.; HODGSON, D.R. 2007. Effect of hypothermia on recovery from general anaesthesia in the dog. Aust. Vet. J. 85(4):158-162. https://doi.org/10.1111/j.1751-0813.2007.00128.x

21. RILEY, C.; ANDRZEJOWSKI, J. 2018. Inadvertent perioperative hypothermia. BJA Educ. (Reino Unido). 18(8):227-233.

https://doi.org/10.1016/j.bjae.2018.05.003
22. REDONDO, J.I.; SUESTA, P.; SERRA, I.; SOLER, C.; SOLER, G.; GIL, L.; GÓMEZ-VILLAMANDOS, R.J. 2012. Retrospective study of the prevalence of postanaesthetic hypothermia in dogs. Vet. Rec. (Reino Unido). 171(15):374. http://doi.org/10.1136/vr.100476

23. RODRIGUEZ-DIAZ, J.M.; HAYES, G.M.; BOESCH, J.; MARTIN-FLORES, M.; SUMNER, J.P.; HAYASHI, K.; MA, E.; TODHUNTER, R.J. 2019. Decreased incidence of perioperative inadvertent hypothermia and faster anesthesia recovery with increased environmental temperature: A nonrandomized controlled study. Vet. Surg. (Estados Unidos). 49(2):256-264.

http://doi.org/10.1111/vsu.13328

24. SANJUÁN, M.; ABAD, E.M.; DE LA FLOR, M. 2011. Termorregulación y manejo perioperatorio. Cir. May. Amb. (España). 16(4):173-190.

25. STEPANIUK, K.; BROCK, N. 2008. Hypothermia and thermoregulation during anesthesia for the dental and oral surgery patient. J. Vet. Dent. (Estados Unidos). 25(4):279-83. https://doi.org/10.1177/089875640802500413

26. THOMAS, J.A.; LERCHE, P. 2017. Anesthesia and analgesia for veterinary technicians. Ed. Elsevier (Canadá). 445p.

27. YANG, L.; HUANG, C.Y.; ZHOU, Z.B.; WEN, Z.S.; ZHANG, G.R.; LIU, K.X.; HUANG, W.Q. 2015. Risk factors for hypothermia in patients under general anesthesia: Is there a drawback of laminar airflow operating rooms? A prospective cohort study. Int. J. Surg. (Holanda). 21:14-17. https://doi.org/10.1016/j.ijsu.2015.06.079

28. YI, J.; LEI, Y.; XU, S.; SI, Y.; LI, S.; XIA, Z.; SHI, Y.; GU, X.; YU, J.; XU, G.; GU, E.; YU, Y.; CHEN, Y.; JIA, H.; WANG, Y.; WANG, X.; CHAI, X.; JIN, X.; CHEN, J.; XU, M.; XIONG, J.; WANG, G.; LU, K.; YU, W.; LEI, W.; QIN, Z.; XIANG, J.; LI, L.; XIANG, Z; PAN, S.; ZHAN, L.; QIU, K.; YAO, M.; HUANG, Y. 2017. Intraoperative hypothermia and its clinical outcomes in patients undergoing general anesthesia: National study in China. PloS One (Estados Unidos). $12(6): 1-13$. https://doi.org/10.1371/journal.pone.0177221 